\title{
The RF Telecommunications System for the New Horizons Mission to Pluto'
}

Christopher C. DeBoy, Christopher B. Haskins, Thomas A. Brown, Ronald C. Schulze, Mark A. Bernacik, J. Robert Jensen, Wesley Millard, Dennis Duven, Stuart Hill

The Johns Hopkins University Applied Physics Laboratory

11100 Johns Hopkins Road

Laurel MD 20723

chris.deboy@jhuapl.edu

Abstract-This paper describes the design and development of the RF telecommunications system for the New Horizons mission, NASA's planned mission to Pluto. The system includes an advanced, low-power digital receiver, and a card-based transceiver implemented within an integrated electronics module. An ultrastable oscillator (USO) provides the precision frequency reference necessary for the uplink radio science experiment. The 2.1 meter high gain antenna is the primary communications antenna, with medium gain and low gain antennas used for wider beamwidths during early operations, cruise-phase and sunpointing mission phases.

The paper will discuss the salient aspects of the system design, including design drivers from mission operations, science, and spacecraft considerations. It will also detail individual subsystems' performance, operational modes (including beacon mode operation), and navigation technology, including the first deep-space flight implementation of regenerative ranging.

\section{TABLE OF CONTENTS}

1. INTRODUCTION .

2. OVERALL SYSTEM

3. UPLINK CARD.

4. DOWNLINK EXCITER CARD.

5. ULTRASTABLE OSCILLATOR

6. ANTENNA SUbSYSTEM

7. NAVIGATION SUPPORT

8. REGENERATIVE RANGING

9. SUMMARY

REFERENCES.

BIOGRAPHIES.............................................. 15

\section{INTRODUCTION}

\section{Mission Description}

New Horizons is NASA's planned mission to Pluto, with a projected launch date in January of 2006. The fly-by mission seeks to characterize the geology and atmosphere of Pluto and its large moon Charon. At launch, the spacecraft follows a heliocentric trajectory to a Jupiter fly-by for gravity assist in 2007, and then settling into a long 8-year cruise to the outermost planet. The baseline encounter date for the Pluto fly-by is July, 2015. An extended mission for the spacecraft would see fly-by's of one or more Kuiper Belt objects, the set of small planetoids of the outer solar system of which Pluto is considered the largest member. Mission trajectories are shown in Figure 1 below, with the three different arrival dates corresponding to launches at different times in the launch window.

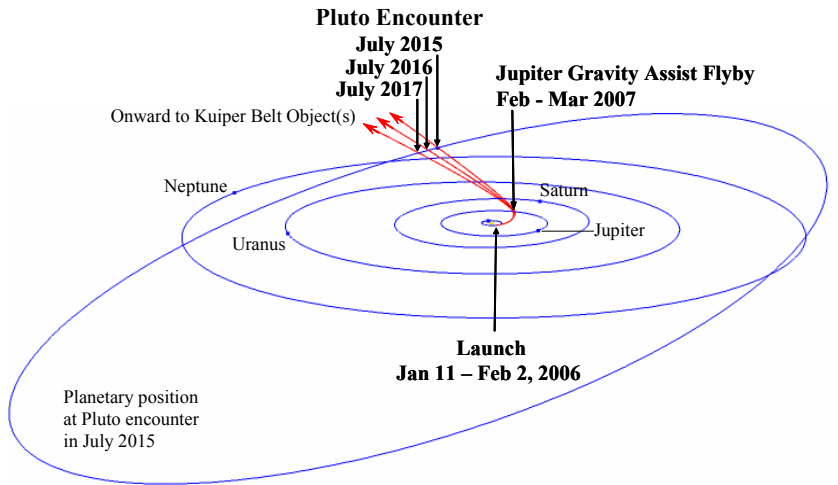

Figure 1 Planned Mission Trajectory

Pluto is on average $39 \mathrm{AU}$ from the Sun, but due to its highly eccentric orbit will be approximately $32 \mathrm{AU}$ from the Earth at encounter $(1 \mathrm{AU}=$ approximately 150 million $\mathrm{km}$.) At that range, the round trip light time to the spacecraft is over 8 hours. Science observations commence in the months

\footnotetext{
${ }^{1} 0-7803-8155-6 / 04 / \$ 17.00 \bigcirc 2004$ IEEE

${ }^{2}$ IEEEAC paper \#1369, Version 4, Updated December 22, 2003
} 


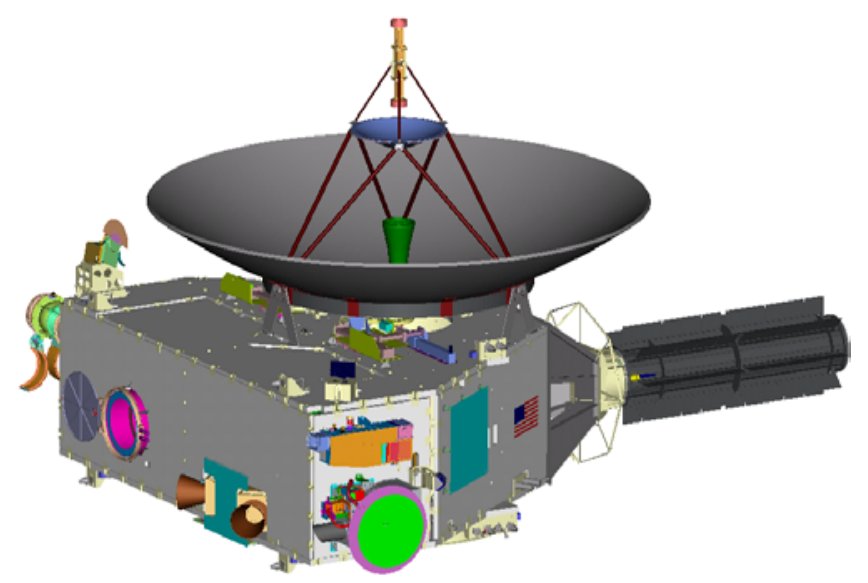

Figure 2 The New Horizons Spacecraft

preceding encounter, with the most intense instrument activity during the several hours before and after closest approach. The scope of the scientific effort includes ultraviolet, visible, and infrared imaging and spectroscopy, particle detection, and radio science. The mission is led by Principal Investigator Dr. Alan Stern at Southwest Research, Inc, and the spacecraft project is led at the Johns Hopkins University Applied Physics Laboratory.

\section{Telecommunications Requirements}

The high level requirements on the RF telecommunications system are to provide uplink and downlink capability throughout the primary and extended mission, to support radiometric range and Doppler tracking for precise navigation, and to incorporate an uplink radio science capability. A minimum post-encounter end-of-playback data rate requirement of $600 \mathrm{bps}$ drives the high gain antenna (HGA) and TWTA designs. The ability to command through the medium gain antenna (MGA) out to $50 \mathrm{AU}$ for the extended mission sets the MGA receive gain.

\section{OVERAll System}

\section{Overview}

A block diagram of the New Horizons RF Telecommunications System is shown in Figure 3.

Integrated Electronics Module (IEM)-Two IEMs are used onboard to house many spacecraft functions, including the command and data handling system, the instrument interface circuitry, the telemetry interface function, the solid state recorder, and the receiver and exciter sections of the telecommunications system, along with the DC-DC converters that power them all. This implementation reduces the overall harness requirement and results in mass and cost savings while offering a flexible platform for future development.

Sets of Uplink, Downlink, and Radiometrics cards are contained within each of two integrated electronics modules (IEMs). The Uplink Card (described in detail in Section 3) provides the command reception capability, as well as a fixed downconversion mode for the uplink radioscience

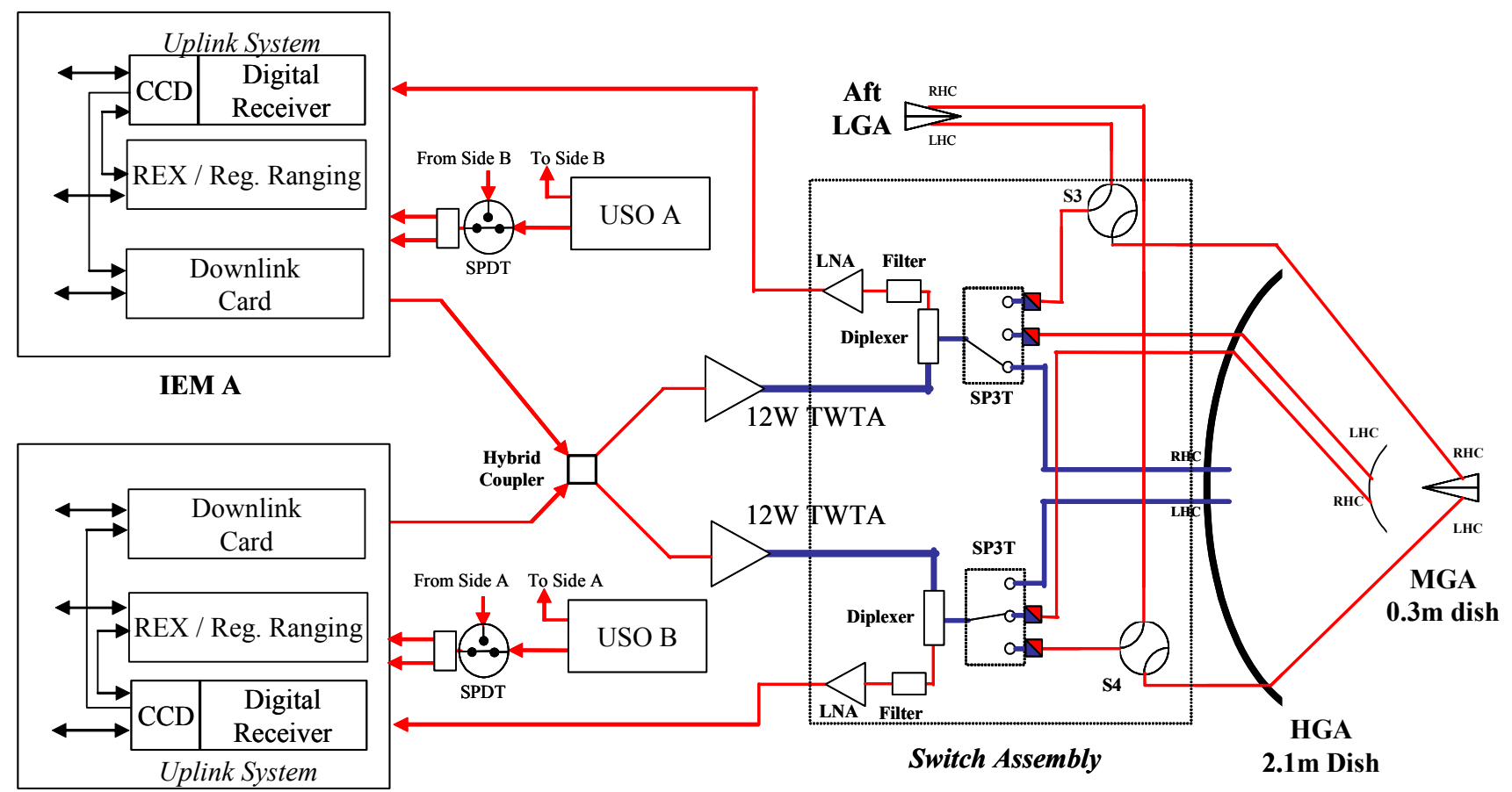

IEM B

Figure 3 RF Telecommunications System Block Diagram

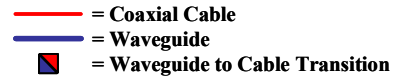


experiment (REX). Since at least one Uplink Card must be powered at all times, this digital receiver's very low power consumption (2.5W secondary) has been an enabling technology for the mission. The Downlink Card (Section 4) is the exciter for the Traveling Wave Tube Amplifiers (TWTAs), and encodes block frame data from the spacecraft Command and Data Handling (C\&DH) system into rate $1 / 6$, CCSDS Turbo-coded blocks. It also calculates and inserts navigation counts into the frame data to support the noncoherent Doppler tracking capability, and is used to transmit beacon tones during cruise periods. The Radiometrics Card contains the REX and Regenerative Ranging Functions.

Ultrastable Oscillators-Two USOs (see Section 5) provide the ultimate frequency reference for the Uplink and Downlink Card local oscillators and clocks. The USOs are cross-strapped with a transfer switch and power splitter to retain redundancy in the Uplink and Downlink Cards in the event of a USO failure.

Hybrid Coupler-A hybrid coupler is connected to the Downlink Exciter outputs and TWTA RF inputs. This enables either exciter to work with either TWTA. In addition to the increased reliability, this gives the Mission Operations team flexibility during downlink events, in that the downlink polarization sense (RHC or LHC) can be changed by powering the appropriate TWTA without having to change IEMs to use the other exciter. It also enables the tantalizing prospect of nearly doubling the postencounter downlink data rate. If both TWTAs can be powered at once (a spacecraft power and thermal margin issue that will be settled during cruise), then, using one exciter, a dual-polarized downlink signal (LHC and RHC) can be transmitted. The Deep Space Network (DSN) has recently tested their single-antenna polarization combining capability and achieved the expected $>2.5 \mathrm{~dB}$ processing gain. Should this mode prove operable, it would provide a $>44 \%$ reduction in the post-encounter playback duration and significant cost savings.

$R F$ Switching and Routing-The RF switch assembly interconnects the antenna suite with the redundant TWTAs and the rest of the communications system. A waveguide diplexer from Ciao Wireless provides the necessary isolation between receive and transmit paths. A bandpass filter prior to the LNA yields more rejection of the downlink signal in the receive path to keep the wideband LNA far from saturation. A SP3T waveguide switch connected to the antenna port of the diplexer enables the RF signals to flow to or from the HGA, MGA, or LGAs. (For the LGAs, an additional SMA transfer switch is used to select the aft or forward antenna.) All connections to the HGA are waveguide to minimize loss, whereas SMA coaxial cables are used for ease of assembly and routing for the MGA and LGA runs.

TWTAs-Two $12 \mathrm{~W}$ X-Band TWTAs manufactured by Thales, Inc., provide the high power RF downlink output The selected power level is a compromised between data rate and beamwidth performance and power dissipation in this power-limited spacecraft.

Antennas - Three antennas are mounted to the forward side of the spacecraft and centered on the spin axis. Spin axis mounting was desirable from both mechanical (greater degree of symmetry about the spin axis) and communications perspectives (high spacecraft spin rates after launch could lead to uplink acquisition and tracking issues if the off-spin-axis distance were too great.) See Section 6 for further discussion on the antenna design.

Uplink Radioscience-The RF Telecommunications System incorporates the REX (Radioscience EXperiment) Instrument. REX seeks to characterize the atmosphere of Pluto and (if one exists) of Charon, and to estimate surface temperatures by recording changes in the received uplink signal at various times during encounter. The hardware specific to REX consists of an analog-to-digital converter and the REX Actel FPGA, and is co-located on the Radiometrics Card with the Regenerative Ranging System (Section 8). A wideband IF output from the uplink receiver is fed to the REX circuitry, and the receiver is commanded to a fixed-conversion mode (i.e., carrier tracking is disabled and all LO's are fixed in frequency.) Any RF input at the appropriate receive frequency is directly downconverted and passed to the REX hardware. Two types of measurements are made. As the spacecraft moves into occultation (the time Pluto blocks the uplink signals from the Earth), samples of the REX filter output are stored to determine the changes in phase and amplitude the uplink signal underwent as it moved through different layers of Pluto's atmosphere. During occultation, the full bandwidth of the REX IF input is integrated, sampled, and later compared to calibrations to determine the effective antenna noise temperature, which can be used to map the physical temperature of the planet.

The REX effort is led by Stanford University, who is responsible for the design of the Actel FPGA. Its inclusion in the RF system mandated tight control of spurious signals in the receiver IF, attention to minimizing the spacecraft system noise temperature (to approximately 200K) and receiver gain variations, and the careful selection of crystal resonators in the USO to achieve the highest level of frequency stability and thus the best performance for REX. 


\section{UPLINK CARD}

\section{Overview}

The New Horizons Uplink Card provides X-band carrier tracking, command detection/demodulation, critical command decoding, ranging tone demodulation, a wideband intermediate frequency (WBIF) channel for use by the Radioscience Experiment (REX) and Regenerative Ranging subsystems, and a fixed downconversion mode for REX. This new uplink card design makes use of digital processing techniques to enhance performance and flexibility, while at the same time achieving considerably lower power consumption than predecessor systems. The Uplink Card (Figure 4) is an assembly consisting of three separate printed circuit boards (PCB) mounted to an aluminum heat sink. On one side, a multilayer, polyimide PCB contains the circuitry required for the RF, IF, analog, and digital portions of the X-band carrier tracking receiver, ranging tone demodulation, and the WBIF channel. Attached to this polyimide $\mathrm{PCB}$ is an RF downconverter board, which consists of a temperature stable microwave substrate, various microstrip circuits, and the circuitry required to complete the first RF downconversion stage in the uplink card. On the other side, a second multilayer, polyimide PCB contains the required circuitry for the command detector unit (CDU) and the critical command decoder (CCD).

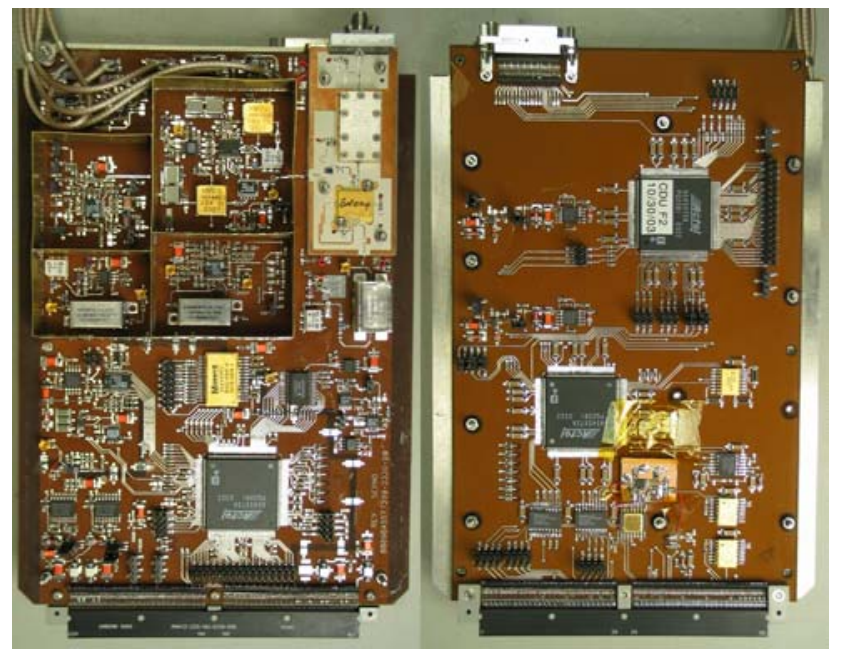

Figure 4- Photographs of Uplink Card

a) digital receiver side, b) baseband side (CDU and CCD)

\section{Design Approach}

The primary RF carrier tracking, command detection, and turnaround ranging channel performance requirements of the digital receiver system are similar to those of previous deep space RF systems, including both the small deep space transponder (SDST) and CONTOUR RF transceiver systems. Several new design requirements led to a new design approach. These new requirements included reduced power consumption, flexibility in the choice of reference oscillator frequencies, and added support for uplink radioscience (REX) and regenerative ranging. The core receiver design uses a classic double-conversion, superheterodyne approach (Figure 5). The aforementioned $\mathrm{RF}$ downconverter board provides several key functions, including power to an external low noise amplifier (LNA) through a microstrip bias tee, band select/image reject filtering, the first downconversion stage, and amplification, tripling, and harmonic filtering of the RF local oscillator (LO). The RF LO is generated at approximately $2474 \mathrm{MHz}$ by a fractional-N synthesizer and routed to the RF downconverter board. The output of the first downconversion stage is the first intermediate frequency (IF), which is approximately $240 \mathrm{MHz}$. The first IF is bandpass filtered and split into two channels, one narrowband and one wideband. The narrowband channel is used for carrier tracking, automatic gain control, and command demodulation, while the wideband channel is used for turnaround ranging demodulation and generation of the WBIF channel to be used by the REX and regenerative ranging subsystems. Both channels each contain a single-chip receiver integrated circuit (IC), which provides the second downconversion stage, automatic gain control (AGC) amplification, and wideband channel quadrature demodulation.

Downconversion to the second IF of $2.500 \mathrm{MHz}$ makes use of an IF LO, which is generated at approximately 242.5 $\mathrm{MHz}$ by an integer-N synthesizer. Upon downconversion to the second IF, separate filtering of the wideband and narrowband channels is achieved via discrete filter circuits external to the aforementioned receiver ICs. The $2.5 \mathrm{MHz}$ narrowband IF (NBIF) channel is processed by a 10-bit, 10 Msps analog-to-digital converter (ADC). The resultant sample data is processed by a field programmable gate array (FPGA), which provides a variety of critical receiver functions. The digital receiver FPGA (DPLL) first demodulates the NBIF to baseband. The baseband data is then processed through the digital portions of the carrier tracking loop, power detection, and AGC system. The DPLL's primary contribution to the carrier tracking loop is in the form of a 20-bit preselect/antialiasing filter and a 64bit loop filter. The output of the loop filter is used to steer the output frequency and phase of a direct digital synthesizer (DDS), which is in turn used as a carrier tracking reference oscillator in the closed loop carrier tracking system. The DPLL's primary contribution to the AGC system is in the form of a power detection circuit and filtering used to drive a digital-to-analog converter (DAC), which is in turn used to generate a control voltage that sets the gain in the analog portions of the receiver system. The digital baseband data is also filtered to select the subcarrier, 


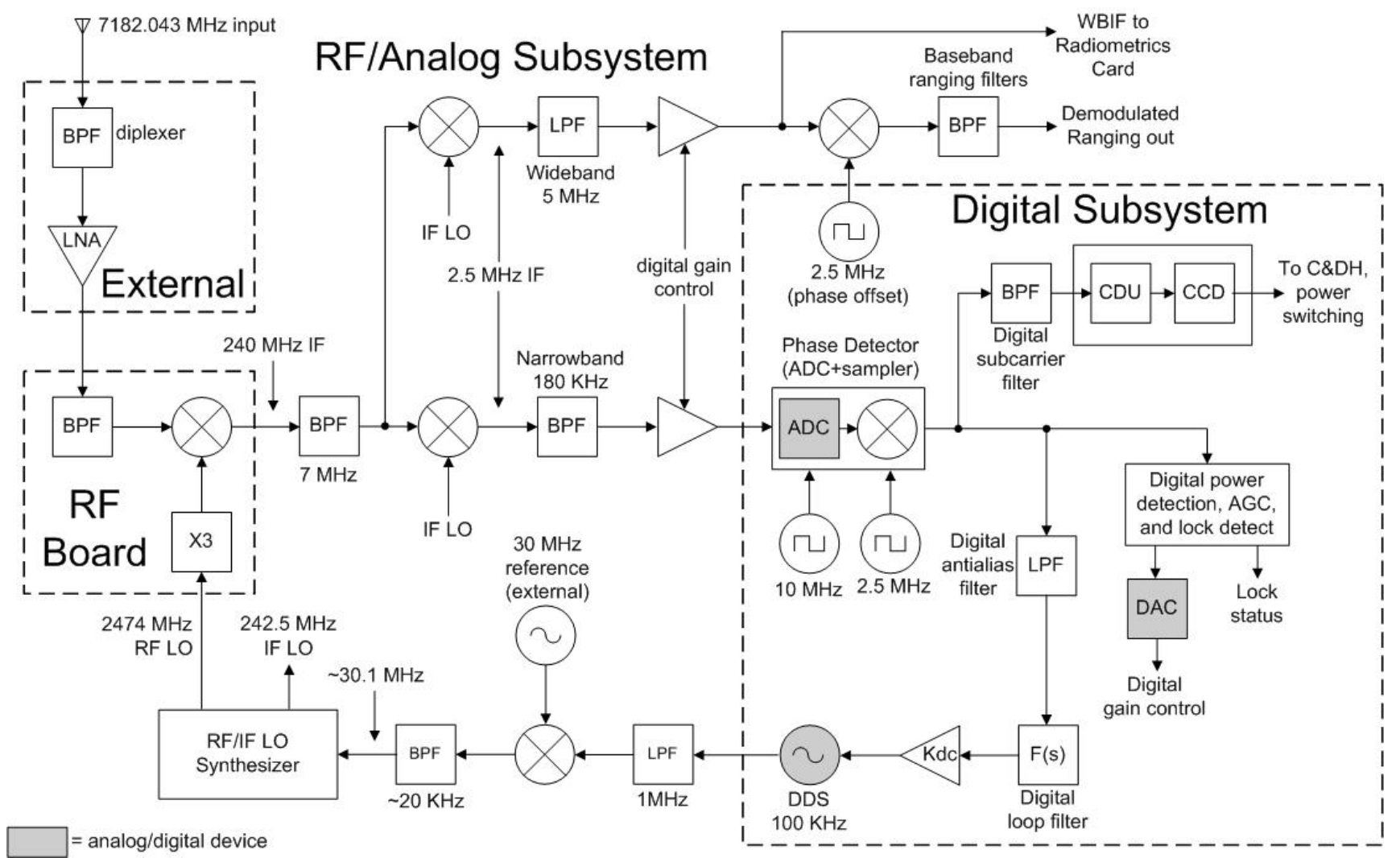

Figure 5 Uplink Card Block Diagram

which is then forwarded to the CDU for data demodulation and command detection. For the New Horizons mission, this subcarrier is binary phase shift key (BPSK) modulated with commands at data rates of $2000,500,125$, and 7.8125 bps. The CDU locks to and tracks the $16 \mathrm{KHz}$ subcarrier and demodulates the command data, passing data and clock over to the CCD. In the CCD, designated critical relay commands are decoded, detected, and immediately sent to the power switching system. The CCD also forwards all commands to the $\mathrm{C} \& \mathrm{DH}$ system.

The 2.5 MHz WBIF channel is buffered and routed to the Radiometrics Card for further processing. The $2.5 \mathrm{MHz}$ WBIF is also demodulated in the quadrature demodulator built into the receiver IC. The resultant baseband channel (or ranging channel) is filtered through several filters designed to limit the noise power in this channel as well as reduce the level of various demodulation products, while at the same time allowing the desired ranging tones to pass through with minimal phase and amplitude distortion. The output of this ranging channel is buffered and routed to the downlink card for modulation onto the downlink X-band carrier. In addition, the ranging channel has the capability to route either the demodulated ranging tones or the regenerated pseudonoise $(\mathrm{PN})$ ranging code produced by the regenerative ranging subsystem to the downlink card.

Carrier acquisition and tracking is provided via a type-II phase locked loop and noncoherent AGC system. Both the
RF and IF synthesized LOs are tuned through the use of a common $30.1 \mathrm{MHz}$ carrier tracking reference clock; this clock is generated by mixing the $30 \mathrm{MHz}$ spacecraft frequency reference with a $100 \mathrm{KHz}$ DDS. The DDS phase and frequency is dynamically tuned by the DPLL carrier tracking system to in-turn tune the RF and IF LOs. An open loop, fixed downconversion mode is required for REX; in this mode, the DDS frequency is set at a fixed value that is reprogrammable during the mission. All clocks and frequency sources in the digital receiver system are referenced to the $30 \mathrm{MHz}$ spacecraft reference oscillator.

\section{Features}

Performance highlights include the following: total secondary power consumption of $2.5 \mathrm{~W}$ (including the integrated on-board command detector unit (CDU) and critical command decoder (CCD)), built-in support for regenerative ranging and REX, carrier acquisition threshold of $-157 \mathrm{dBm}$, high RF carrier acquisition and tracking rate capability for near-Earth operations $(2800 \mathrm{~Hz} / \mathrm{s}$ down to $100 \mathrm{dBm}, 1800 \mathrm{~Hz} / \mathrm{s}$ down to $-120 \mathrm{dBm}, 650 \mathrm{~Hz} / \mathrm{s}$ down to $-130 \mathrm{dBm})$, ability to digitally tune to any X-band RF channel assignment (preprogrammed on Earth for this mission) without the need for analog tuning and tailoring, use of an even $30.0 \mathrm{MHz}$ ultrastable oscillator (USO) as a frequency reference, a noncoherent AGC system, and best lock frequency (BLF) telemetry accuracy to $0.5 \mathrm{~Hz}$ at Xband and BLF settability plus stability error $<+/-0.1 \mathrm{ppm}$ 
with zero temperature effects (all relative to USO frequency).

These results highlight the major performance parameters of the operational and functional RF uplink system. One of the key benefits of moving to a more-digital system is increased operational flexibility. Once in place, successive design iterations in future missions may include in-flight reconfiguration of RF channel assignments, and carrier tracking loop optimization for near-Earth, deep space, and interstellar operational modes. A secondary benefit is the ability to leverage from increasing gate array densities and processing unit speeds, thus contributing to further mass, size, and power savings. Finally, further reduction in hardware assembly steps due to lower parts count and fewer solder connections increases the reliability of these systems.

\section{DOWNLINK EXCITER CARD}

\section{Downlink Card Overview}

The New Horizons Downlink Card provides an X-band exciter output to be delivered through a hybrid coupler to the spacecraft TWTA for transmission. This output consists of the carrier (either unmodulated or residual) and phase modulation for the telemetry, ranging, or beacon modes of operation.

The Downlink Card (see picture in Figure 6 and block diagram in Figure 7) is an assembly consisting of three separate printed circuit boards (PCB) mounted to an aluminum heat sink. On one side, a multilayer polyimide PCB contains the synthesizer and signal conditioning circuitry, along with a temperature-stable microwave substrate containing S- to X-band multipliers and phase modulators. On the reverse side, a second multilayer polyimide PCB contains the digital circuitry for the PCI interface, modulation control, noncoherent Doppler tracking counters, CRC calculation and Turbo encoding functions.

\section{Design Approach—Analog Side}

In this transceiver system, the carrier signal is generated by a PLL synthesizer using the on board $30 \mathrm{MHz}$ USO reference. Two output center frequencies are possible, designated primary and auxiliary. The primary or auxiliary frequency is selected by controlling the divisors in the synthesizer's parallel load inputs. This capability permits the downlink carrier to be switched between carrier frequencies depending on the state of the uplink carrier lock indicator (allowing the downlink to provide 1 bit of information on the uplink status.) If this coherent simulation mode is disabled the downlink carrier is always at the primary center frequency. The synthesizer output signal at S-Band is mixed with the $30 \mathrm{MHz}$ USO reference to reduce the potential perturbation of the PLL loop by the desired signal and harmonics as it is multiplied to X-band. The carrier is modulated at $4 \mathrm{GHz}$, with a pair of phase modulators, one for the ranging signal from the uplink card, the other for the telemetry data. The ranging signal is processed by an AGC amplifier stage, which also provides a means of disabling the ranging signal. HBT amplifiers were initially considered for the $4 \mathrm{GHz}$ and $8 \mathrm{GHz} \mathrm{RF}$ amplifier stages, but were ultimately changed to GaAs MMIC due to reliability concerns over the extended operational life required for the mission.

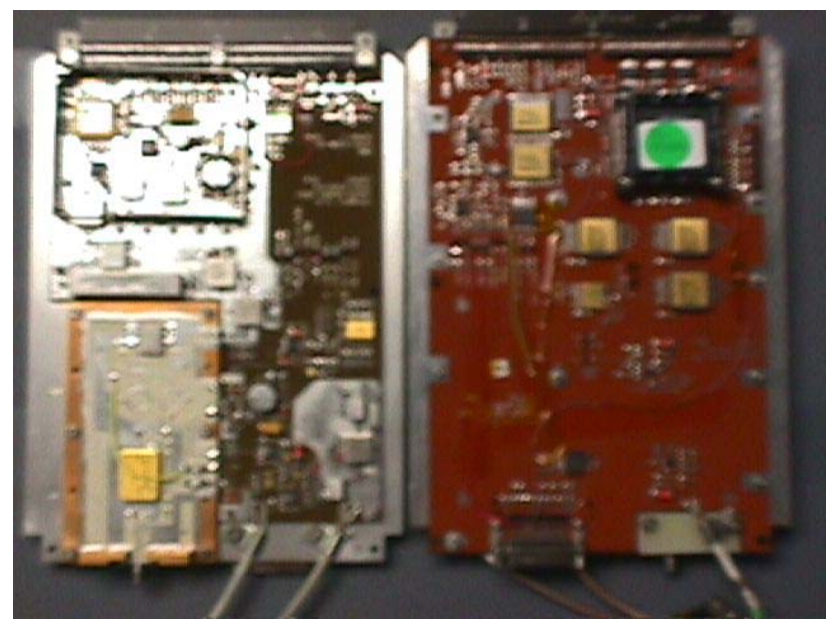

Figure 6 - Photographs of Downlink Card Left, Analog side, Right Digital side

\section{Design Approach—Digital}

The majority of the Downlink Card's digital design is contained within a single Actel FPGA, an RTSX72S. Additional circuitry consists of the $5.3 \mathrm{kHz}$ Doppler tracking signal filter, which is implemented in op-amps, and the PROMs and RAMs and interface circuitry.

The FPGA design is implemented in VHDL. Its primary function is to Turbo-encode frame data blocks from the C\&DH system. The encoder uses the interleaving tables stored in the external, anti-fuse PROMs to create the coded symbol streams, and calculates and inserts a CRC for each frame. The design also includes a PCI interface that enables frame data transfer and downlink bit rate and mode selection. Also included are the noncoherent Doppler tracking counters, including sub-frame count interface and control, CRC calculation and insertion (for each frame), interleaving and turbo encoding, and a subcarrier modulator. Generally for low data rates, this modulator places NRZ line coded symbols onto a $25 \mathrm{kHz}$ subcarrier. Other functional blocks include PN code generators for BER testing, implementation of test modes, and MET (Mission Elapsed Time) counters to accurately record the 


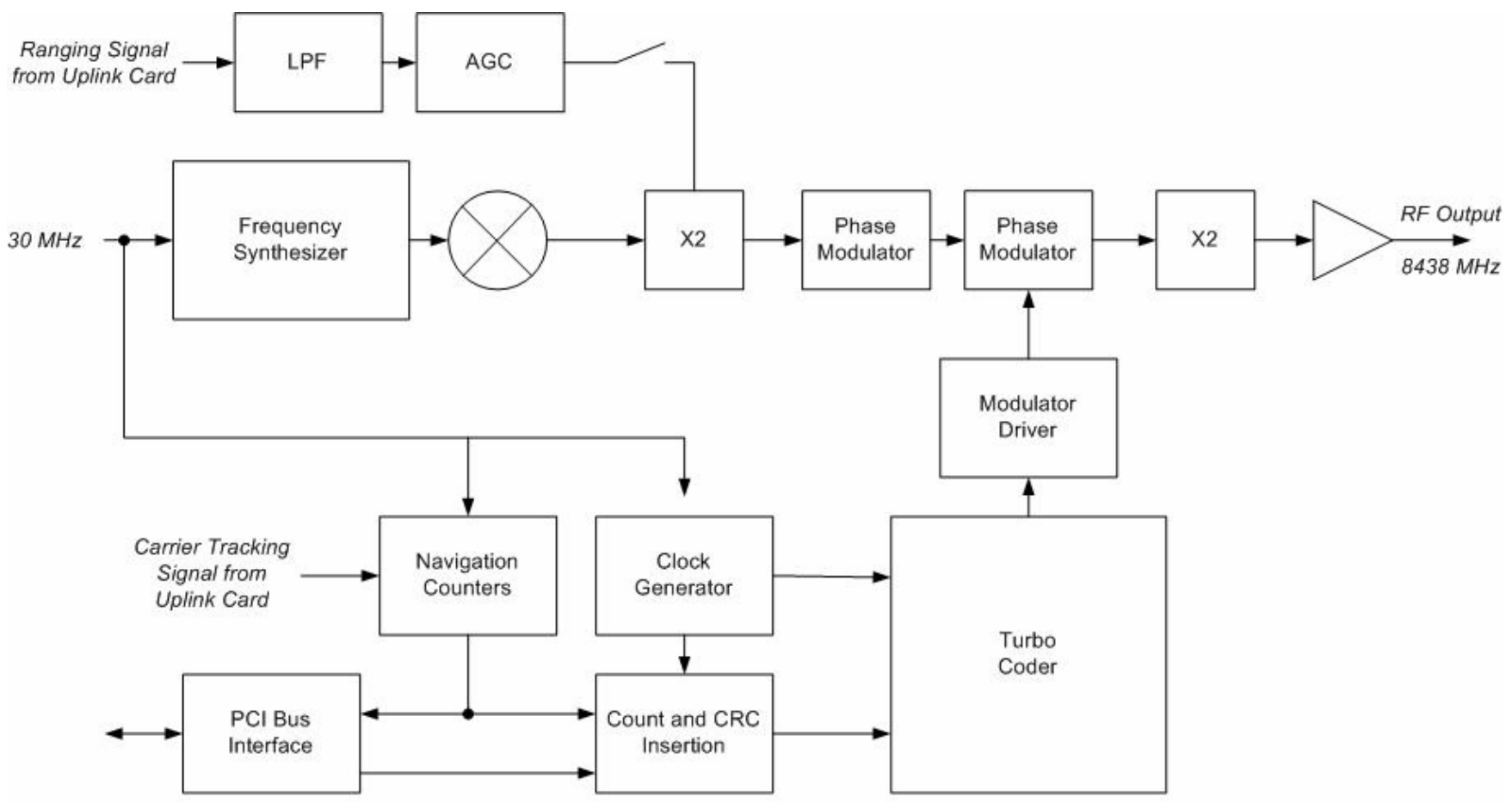

Figure 7 Downlink Card Block Diagram

start time of a frame transmission. (This result is included in telemetry in the next downlinked frame.)

A 16 bit control register is used to provide 65532 possible downlink data rates, from a minimum of $6.3578 \mathrm{bps}$ to a maximum of $104.167 \mathrm{kbps}$. The relation between control word setting $\mathrm{n}$ and the data rate is

$$
\text { BitRate }=\frac{5 \mathrm{MHz}}{12(n+1)}
$$

where $3 \leq \mathrm{n} \leq 65536$. The fine spacing of data rates about the expected post-encounter playback rate of $1 \mathrm{kbps}$ gives the mission operations team a great deal of flexibility to take advantage of late improvements in the space segment and ground segment system performance. To bound the pre-flight test time required, a subset of these data rates will form the baseline for ground test.

Since the CRC must be calculated and inserted before the data interleaving and turbo encoding, it is necessary to pipeline data frames, with the next frame being encoded while the current frame is being transmitted. This requires the use of two RAM frame buffers. In addition, to avoid possible underflow when operating at maximum rates (primarily during test), a third null buffer was included and filled with dummy data should a frame be completely transmitted before the next frame has been encoded.

The Downlink Card can also be configured to support Beacon Mode operations. In this mode, a single square wave tone is phase-modulated onto the selected carrier. The frequency of the beacon tone is determined by the setting of the 16bit data rate control register: the tone frequency is the symbol rate that corresponds to the commanded data rate (i.e., the tone frequency is $6 \mathrm{x}$ the selected data rate.) This gives the software and mission operations teams flexibility in defining tone frequencies and minimizes the impact of this new capability to the existing card design. The hardware in the DSN supports up to 4 separate defined tone frequencies that can be used. By placing tones on either carrier, 8 different beacon settings can be selected to provide various levels of information.

\section{Ultrastable OSCILlator}

\section{Overview}

The New Horizons Ultrastable Oscillator (USO) is a critical component of the RF telecommunications system and the mission itself. It provides a stable, 30 $\mathrm{MHz} \quad$ frequency reference for the Uplink and Downlink Cards' frequency synthesizers, and the ultimate frequency reference necessary for the uplink radio science experiment.

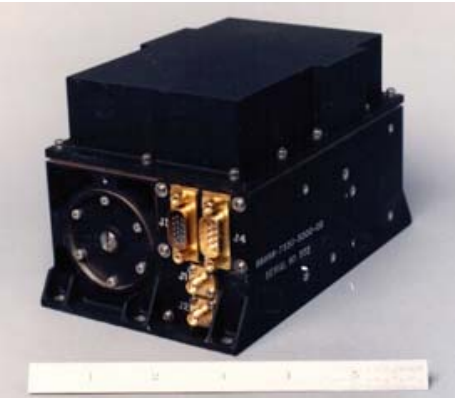

Figure 8 Ultrastable Oscillator Assembly 
The USO is a sophisticated precision assembly consisting of over 200 electronic components and many mechanical parts (see photo in Figure 6). Its architecture builds on proven heritage designs developed at APL over the last 30 years, and flown recently on such missions as Mars Observer, Cassini, GRACE, and Gravity Probe B. Fundamentally, it is a pristine version of an ovenized crystal oscillator (OCXO). We use carefully selected, $3^{\text {rd }}$ overtone, SC-cut crystal resonators and maintain them at constant temperature to yield excellent frequency stability (shortterm to better than 1 part in $10^{13}$ ) and low noise performance. The significant performance improvement over industry grade OCXOs relies on a cylindrical oven design and very uniform heating which results in very small temperature gradients within the crystal resonator blank. A high-gain thermal control loop keeps the crystal resonator temperature stable to within several thousandths of a degree Celsius over the entire operating temperature range of the USO. Crystal resonators for flight will be carefully selected among twenty units for the best aging and short-term frequency stability.

\section{Functional Description}

The block diagram of the USO is shown in Figure 7. The USO consists of six polyimide printed wiring boards populated with military-grade, high-reliability passive and active components:

$A 1$ and $A 2$ - The A1 board interfaces the electronic circuitry with the resonator, and consists of the oscillator front end, an automatic gain control (AGC) circuit, and a high input impedance buffer amplifier. AGC sets the oscillator start-up loop gain and resonator drive level, which will be carefully tailored for optimum combination of aging performance and short term stability. The A2 board generates the temperature control signal to drive a heater that keeps the oven temperature of the crystal resonator constant. Since both the A1 and A2 boards contain temperature-sensitive circuitry, they are part of the temperature control assembly operating at a fixed temperature.

$A 3$ and $A 4$ - These boards multiply, filter, and amplify the $5 \mathrm{MHz}$ resonator output to the $30 \mathrm{MHz},+10 \mathrm{dBm}$ signal required in the rest of the RF system. High-Q circuits and multiple transmission zeros in the output stage result in harmonics at levels less than $-60 \mathrm{dBc}$ and subharmonics at levels of less than $-80 \mathrm{dBc}$.

A5 and A6- The A5 board collects and conditions the USO telemetry (voltages and temperatures) for output, while the A6 board cleanly regulates the $22 \mathrm{~V}-35 \mathrm{~V}$ input bus voltage down to $15 \mathrm{~V}$.

\section{Performance Highlights}

The resolution of the New Horizons uplink radioscience experiment depends upon the excellent frequency stability of the USO over the duration of the Earth occultation event at Pluto. Short-term frequency stability (Allan deviation) at $1 \mathrm{~s}$ and $10 \mathrm{~s}$ are specified at $3 \times 10^{-13}$ and $2 \times 10^{-13}$, respectively, with goals of sub- $1 \times 10^{-13}$ performance. At this writing, with roughly half of the lot of crystal resonators tested, the best Allan deviation (10 s) achieved is better than $1 \times 10^{-13}$.

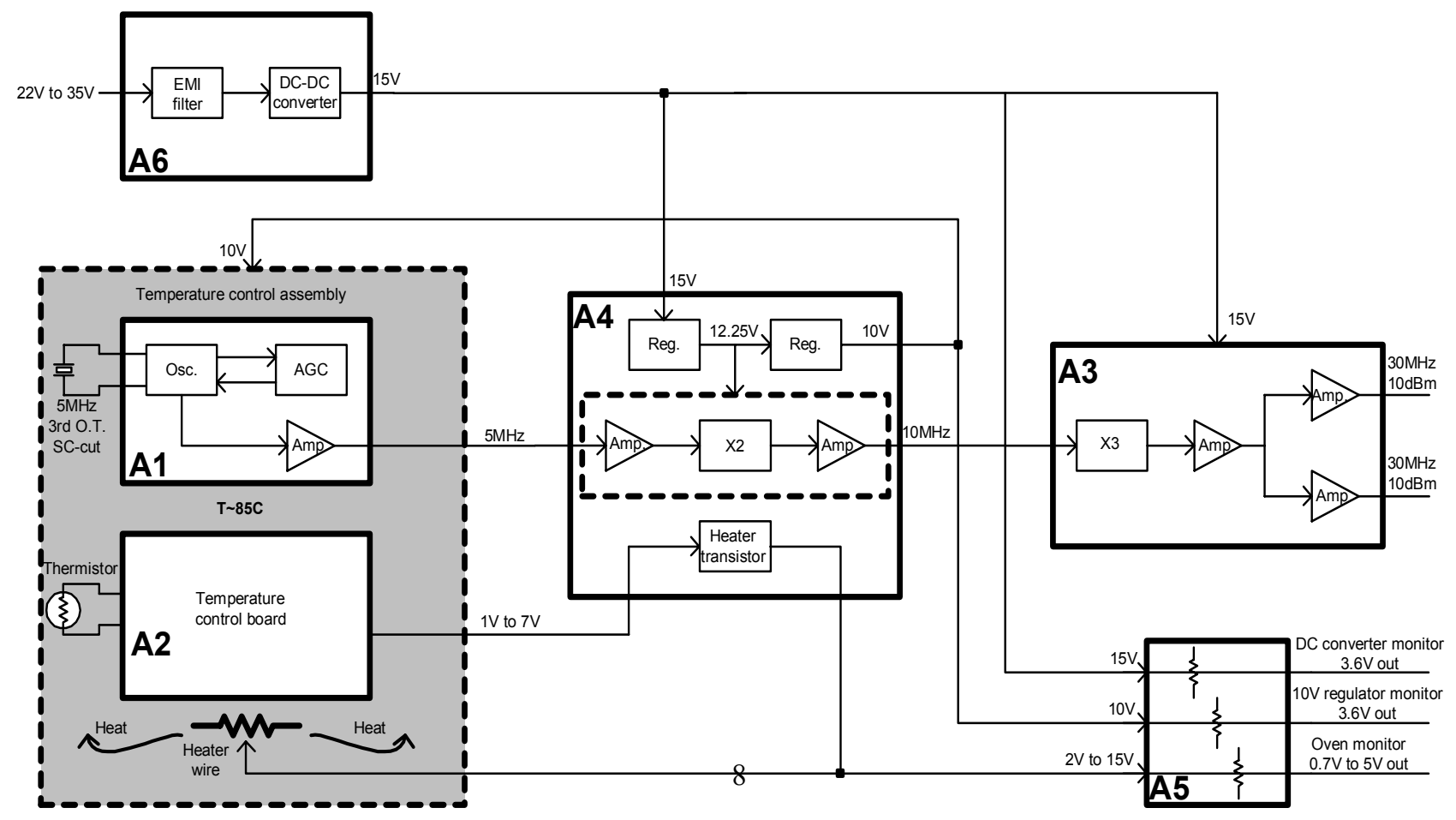

Figure 9 Ultrastable Oscillator Block Diagram 
A summary of other relevant performance measures is tabulated below.

\begin{tabular}{|c|c|}
\hline Parameter & Specification \\
\hline Output Frequency & $30 \mathrm{MHz}$ \\
\hline $\begin{array}{l}\text { Output Power } \\
\text { (into } 50 \Omega \text { ) }\end{array}$ & $\begin{array}{l}+10 \mathrm{dBm}+/-1 \mathrm{~dB} \\
\text { (dual output) }\end{array}$ \\
\hline Aging Rate & $\begin{array}{lll}<1 \times 10^{-11} & \text { per } & \text { day } \\
\text { (goal) }\end{array}$ \\
\hline DC Power & $<3.5 \mathrm{~W}$ steady state \\
\hline SSB Phase Noise & $\begin{array}{l}<-125 \mathrm{dBc} . \mathrm{Hz} @ 100 \\
\mathrm{~Hz}\end{array}$ \\
\hline $\begin{array}{l}\text { Frequency stability } \\
\text { over temperature }\end{array}$ & $<1 \cdot 10^{-12}$ per ${ }^{\circ} \mathrm{C}$ \\
\hline Temperature range & $-25^{\circ} \mathrm{C}$ to $\left.+65^{\circ} \mathrm{C}\right)$ \\
\hline Mass & $<1500$ grams \\
\hline
\end{tabular}

Table 1 USO Performance Specifications

\section{AnTENNA SUBSYSTEM}

\section{Overview}

The NH antenna system is designed to support low- and high-data-rate communications from both a spinning and three-axis stabilized platform. During most of the mission, the spacecraft is spin stabilized (for example, the long cruise to Pluto and during the science playback post-encounter.)

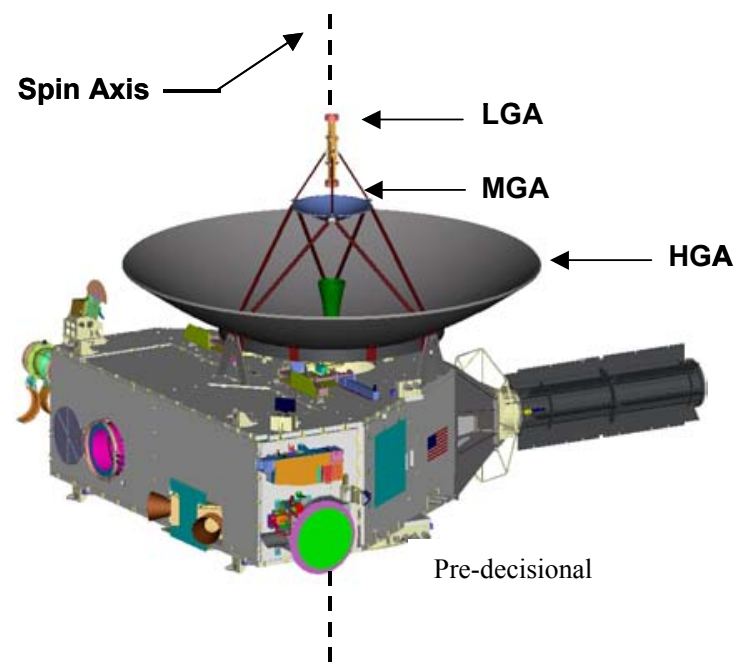

Figure 10 Forward Antenna Stack on New Horizons

Thus, the patterns of the antennas are symmetrical and positioned away from the spacecraft structure to prevent blockage and interference nulls. The resulting design is a stack arrangement of antennas (HGA, MGA, LGA) on the forward end of the observatory as shown in Figure 10. This configuration is optimum because it satisfies all of the antenna field-of-view requirements and the stringent mass limitations imposed by the mission. It is also based on requirements that the forward low gain antenna be located sufficiently near or ideally on the spin axis and that the LGA will provide communications coverage $\pm 90^{\circ}$ from the spin axis, which limits other locations for the MGA and HGA. Not visible in this figure is the aft low gain antenna, which is also placed on the spin axis beneath of the spacecraft.

\section{Low Gain Antennas}

The low-gain antennas on each end of the observatory are slightly different. The directional pattern of the aft LGA is narrower compared to the forward LGA because the aperture of the aft LGA is only 3 inches above the plane of the third-stage interface ring. This means that the field-ofview for the forward low gain antenna is wider so a lower gain (i.e. broader pattern) is used. Both low gain antennas are derivatives of open-ended waveguide antennas. However, RF chokes are added on the outside of the waveguide walls to shape the patterns of each LGA. The chokes on the aft antenna are in the plane of the waveguide aperture and are designed to increase its gain to reduce pattern ripple in the coverage region due to the spacecraft structure reflections. The chokes on the forward antenna are recessed from the aperture, as shown in Figure 11, and strategically positioned to improve the front-to-back ratio [1] to minimize any back-scattering from the MGA or HGA.

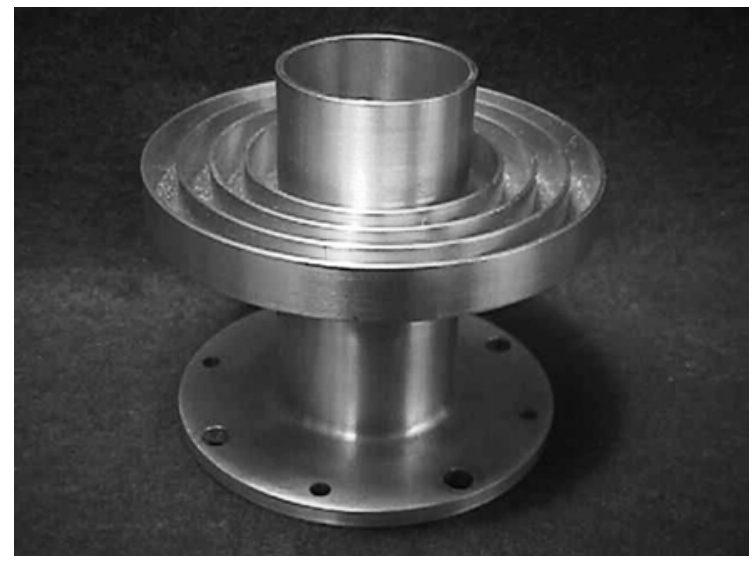

Figure 11 Forward LGA

In both cases, the chokes improve the axial symmetry of the radiation pattern, which is important because the spacecraft is spin stabilized. The downlink patterns of the forward and aft low-gain antennas are shown in Figure 12.

\section{Medium Gain Antenna}

The stack arrangement on the forward side of the observatory shows the medium gain antenna (MGA) 
positioned below the forward LGA. The MGA is a 14.5inch diameter, circular symmetric parabolic reflector, illuminated with a horn that is identical to the aft LGA.

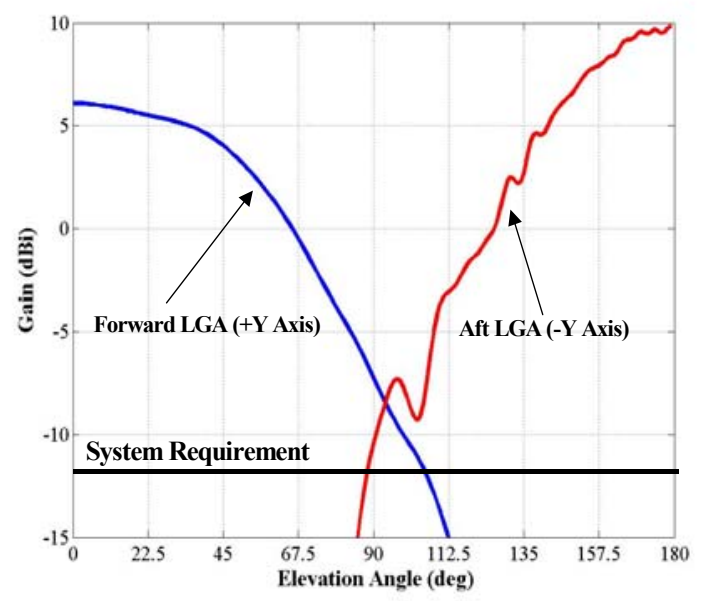

Figure 12 LGA Antenna Patterns

This horn is ideal as a feed because its blockage area is small, it possesses excellent front-to-back ratio, and the position of the phase center is well defined at the uplink and downlink frequencies. A 3/8-inch strut supports the feed horn approximately 6 inches from the reflector vertex. The diameter of the MGA is optimized for uplink and downlink gain at 4 degrees from bore-site.

\section{High Gain Antenna}

A highly efficient 2.1-meter parabolic reflector antenna (the HGA) is required to support the minimum 600 bps 36-AU post encounter return link. This diameter reflector was selected after the NH antenna team performed a detailed alignment budget, which included effects due to thermal distortions on the spacecraft bus and the antenna system, dimensional tolerances, measurement knowledge of the antenna bore sight, ground station pointing errors, power margins, and minimum antenna gain. The alignment analysis showed that the HGA system could be accurately aligned to within $0.2^{\circ}$ of the spacecraft spin-axis. An additional $0.1^{\circ}$ misalignment with the DSN ground station pointing due to ephemeris is allowed for a total alignment budget of $0.3^{\circ}$. A larger sized reflector antenna can be used on spin-stabilized spacecraft with enhanced definition, knowledge, and control of variables in the alignment budget, which increases the engineering cost of the spacecraft. The 2.1-meter reflector is attractive because it is lightweight, meets the communication requirements, and is relatively easy to handle during spacecraft integration.

A communications link analysis established that the HGA is required to provide a minimum antenna downlink gain of 42 $\mathrm{dBic}$, as shown in Figure 13, for angles within $\pm 0.3^{\circ}$. The gain requirement could be achieved with a standard Cassegrain reflector design but the margin at these angles is small, as shown. A key RF design goal for the NH mission is to maximize coverage over these angles, which is accomplished here by modifying the shape of both reflecting surfaces until the aperture fields (amplitude \& phase) are uniform.

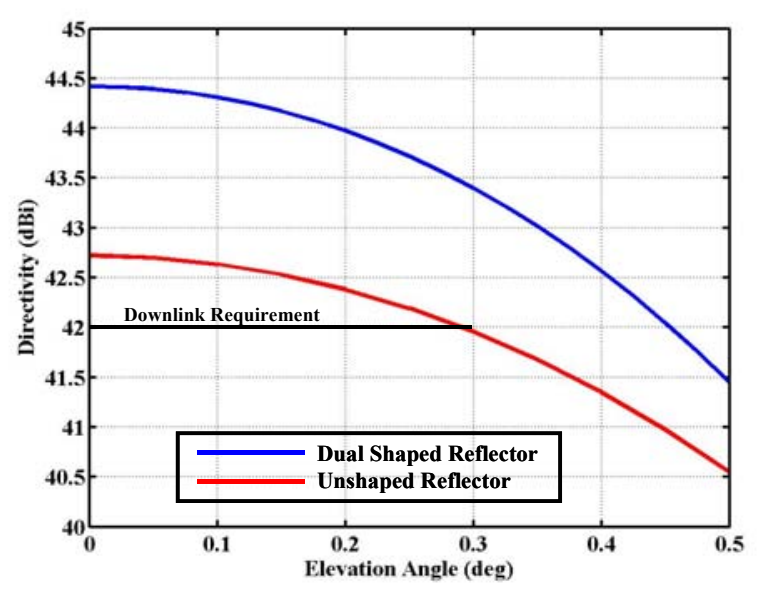

Figure 13 HGA Downlink Gain

\section{Mechanical Design}

The mechanical design of the New Horizons High Gain Antenna is dictated by the $-200^{\circ} \mathrm{C}$ to $+80^{\circ} \mathrm{C}$ operational temperature range the antenna system will be exposed during the life of the mission. The cryogenic operational temperature is expected for the Pluto and $\mathrm{KBO}$ encounters, while the upper temperature of $+70^{\circ} \mathrm{C}$ occurs early in the mission, when maneuvers may require pointing the HGA directly at the sun. In order to meet critical science and communication mission requirements, the mechanical alignment of the HGA bore sight to the spacecraft spin axis must be maintained to a high degree of accuracy over this temperature range. Composite materials are base-lined for the HGA mechanical design, in order to realize these requirements. While more costly and complex in application, composite materials offer a dimensionally stable mechanical design over the expected temperature range as well as a high stiffness and low mass solution required for the launch loads. The fabrication of the HGA reflector utilizes a 0.75 -inch thick composite sandwich layup with a Korex honeycomb core. The secondary reflector assembly consists of a MGA and HGA sub-reflectors and is a composite laminate shell lay-up. Three (3) composite struts with Invar fittings are used to support the MGA feed horn and a six (6) strut configuration, similar to Voyager and Cassini designs, positions and centers the sub-reflector with the HGA main reflector and feed horn. 


\section{NAVIGATION SUPPORT}

The downlink frequency from the New Horizons transceiver is derived from the USO, as discussed previously, but even this excellent frequency reference is not sufficient to support precise and accurate Doppler velocity measurements at the target level of $0.1 \mathrm{~mm} / \mathrm{s}$ throughout the mission. In order to remove the impact of frequency drift and bias in the reference oscillator, New Horizons has implemented the noncoherent Doppler velocity measurement approach [2] which was successfully demonstrated in 2002 during the demanding Earth orbit phase of the CONTOUR mission [3].

\section{Noncoherent Doppler Tracking}

The noncoherent Doppler approach is based on a comparison of the spacecraft frequency reference with the uplink frequency received by the spacecraft. This comparison information is included in telemetry. The ground stations measure the downlink Doppler with the same setup as would be used if the spacecraft communications system was coherent. The telemetered frequency comparison information is used in a software step that converts the observed Doppler frequency to that which would have been observed had the spacecraft had carried a coherent transponder. Doppler navigation is then unaffected by the use of a transceiver.

Accurate alignment in time of the measurements made on the ground and on the spacecraft is critical to meeting the mission navigation requirements. This is accomplished by relating both sets of measurements to the transfer frame boundaries. The frequency comparison made on the spacecraft is applicable on the ground after a one-way lighttime delay and the use of transfer frames to carry the timing information naturally accounts for this delay. No knowledge of the light-time from the orbit determination is necessary to perform the Doppler correction. Only the observed Doppler frequency and the telemetered data are required.

The onboard frequency comparison is accomplished through the use of two 16-bit counters and a small amount of associated logic. These counters are included on the downlink card. The counters are sampled soon after the start of each transfer frame and the results are placed into the secondary frame header of the following frame. This delay is necessary because the CRC for the frame has already been computed prior to start of transmission of the frame, as discussed above.

While one set of counter measurements is made after the start of every frame at all data rates and at both frame length, this data alone would be insufficient to support the orbit determination at the lower data rates where the frame durations can be several minutes long. Therefore, the counters are sampled within the frame and these samples are collected by the C\&DH system for inclusion in the telemetry.

The implementation of the navigation counters is included in the Actel FPGA that includes the Turbo encoder. No additional components are necessary. The framer is also incorporated into this FPGA, so the inclusion of the counter data into the secondary frame header is easily accomplished.

All of the counter measurements, both those that follow the frame start and those that are made within the frame, are available to the C\&DH system through the PCI interface.

Laboratory testing at APL and compatibility testing with DSN equipment have demonstrated that the use of the noncoherent Doppler approach will meet the mission Doppler velocity requirements.

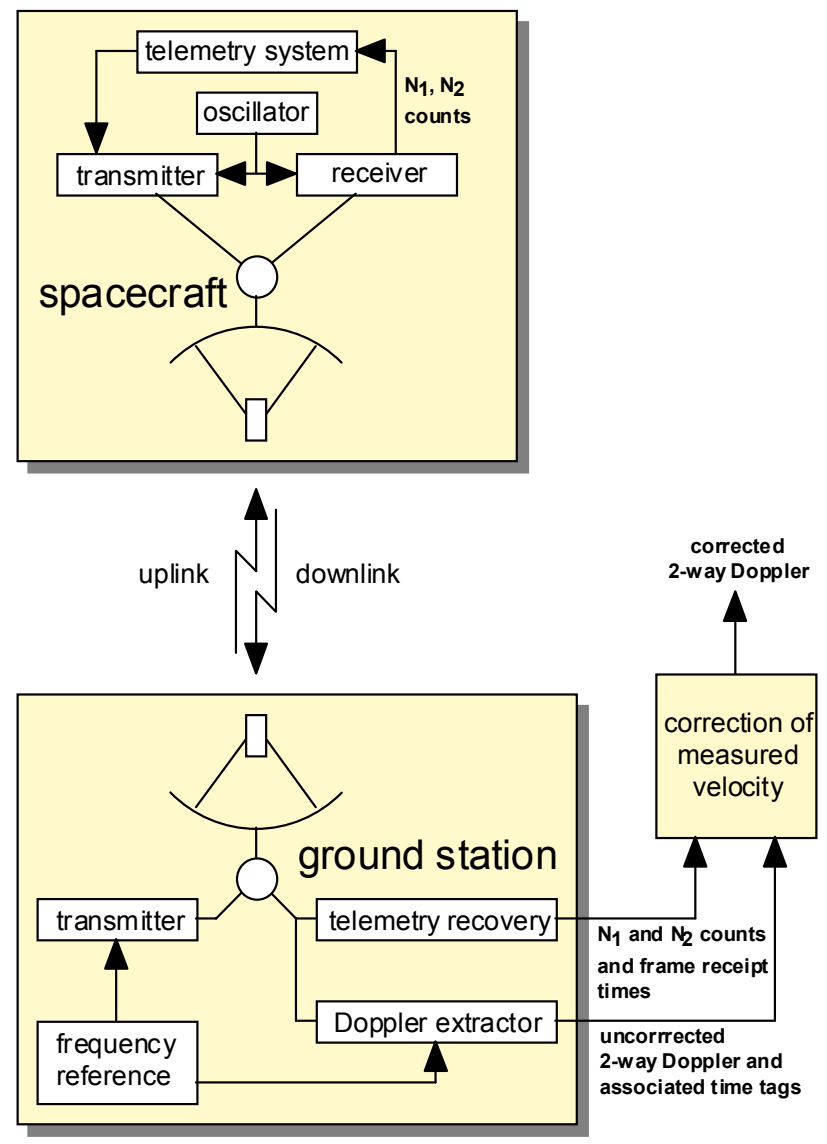

Figure 14 Noncoherent Doppler Tracking

\section{Ranging}

The use of a noncoherent transceiver does impact ranging measurements since the DSN expects the turnaround ranging tones to have a two-way Doppler shift, as they 
would in a coherent transponder. For New Horizons, as it was for CONTOUR, the uplink frequency will be adjusted to remove the one-way Doppler on the uplink signal. Ranging measurements will then be made as normal since the downlink carrier frequency and the ranging tone will both experience a one-way downlink Doppler shift. This approach requires only an approximate knowledge of the spacecraft velocity and frequency reference and does not require special spacecraft hardware. The Doppler tracking counts, along with the other metrics in the tracking process, enable the USO to be tracked in real time so that any unexpected changes in USO frequency (e.g., due to spin up or spin down) can be accounted for.

\section{REgENERATIVE RANGING}

Metric tracking of interplanetary spacecraft is normally accomplished by sending a phase-modulated RF carrier from one of three available DSN transmitter sites, receiving and retransmitting that signal with a wide bandwidth transponder located in the spacecraft, receiving the transponded signal at a DSN receiver site, and processing the received signal in a way that allows the 2 -way range and range-rate (or Doppler) data for the spacecraft to be measured. The range-rate measurements are derived from the Doppler shift of the received carrier whereas the range measurements are derived from the time delay of the modulation component of the received signal. The modulation signal has traditionally been a sequence of sine or square-wave tones with frequencies ranging from $1-\mathrm{MHz}$ down to a few Hertz. The time delay measurements derived from each tone can be combined in a way that allows the ambiguity interval of the composite measurement to be a relatively large fraction of a second. When these measurements are multiplied by the speed of light and combined with the dynamics of motion equations provided by classical or relativistic physics, the 2-way range values can be unambiguously determined.

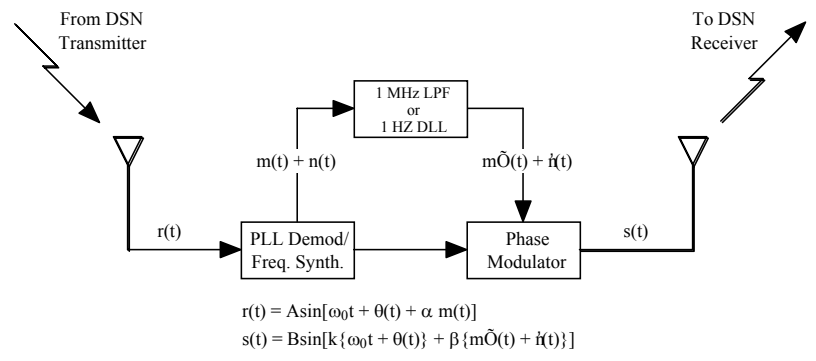

Figure 15 Signal Processing for Spacecraft Tracking

The signal processing that occurs in a conventional transponder is shown in Figure 15. A phase-locked loop (PLL) locks to the received carrier $\omega_{0} t+\theta(t)$ and provides: (a) a synchronously related carrier at another frequency $k\left[\omega_{0} t+\theta(t)\right]$ which serves as the carrier for the downlink transmitter, and (b) the modulation signal $\mathrm{m}(\mathrm{t})$ which was phase modulated onto the uplink carrier. However, since the amplitude of the received uplink carrier is typically quite small, the recovered modulation signal is usually accompanied by a Gaussian noise term $n(t)$ whose $1 \sigma$ amplitude may be equal to or greater than the amplitude of $\mathrm{m}(\mathrm{t})$. A low-pass filter (LPF) is used to reduce the amplitude of the noise as much as possible, but the filter must also be wide enough to pass the highest modulation frequency of $1 \mathrm{MHz}$ with no more than a modest amount of attenuation. As a result, a considerable amount of noise is passed on to the downlink modulator.

The regenerative ranging circuit (RRC) provides a way of substantially reducing the amount of noise that is passed on to the downlink modulator. In this case the $1 \mathrm{MHz} \mathrm{LPF}$ is replaced with a delay-locked loop (DLL) that generates an on-board replica $\mathrm{m}^{\prime}(\mathrm{t})$ of the received modulating signal $m(t)$ and adjusts the timing of $m^{\prime}(t)$ to match the delay of $m(t)$. The effective bandwidth of this process will be approximately $1 \mathrm{~Hz}$ as opposed to the $1 \mathrm{MHz}$ bandwidth of the transponder. The amount of noise passed on to the downlink modulator will consequently be reduced by several orders of magnitude. This regenerative ranging technique was first developed for deep space applications by Berner, et al., at JPL [4], and New Horizons has adopted their standard for implementation into the flight system. New Horizons will be the first mission to test regenerative ranging in flight.

The modulating signal best suited for DLL tracking is a pseudorandom noise (PRN) code defined in [C]. This code consists of a repeating sequence of $\mathrm{N}=1,009,470$ binary chips clocked at a rate $f_{\text {chip }}$ that is proportional to the $R F$ carrier frequency. For the New Horizons mission this frequency will be $\mathrm{f}_{\text {chip }}=2,069,467.087$ chips $/ \mathrm{sec}$. The corresponding code repetition interval will be $\mathrm{P}=\mathrm{N} / \mathrm{f}_{\text {chip }}=0.487,792,247$ seconds. The ranging code is actually a composite code calculated from a set of six periodic binary component generators according to the equation

$$
\mathrm{C}(\mathrm{i})=\mathrm{C}_{1}(\mathrm{i}) \mid\left[\mathrm{C}_{2}(\mathrm{i}) \& \mathrm{C}_{3}(\mathrm{i}) \& \mathrm{C}_{4}(\mathrm{i}) \& \mathrm{C}_{5}(\mathrm{i}) \& \mathrm{C}_{6}(\mathrm{i})\right]
$$

The length of each component will be $\mathrm{N}_{1}=2, \mathrm{~N}_{2}=7, \mathrm{~N}_{3}=11$, $\mathrm{N}_{4}=15, \mathrm{~N}_{5}=19$, and $\mathrm{N}_{6}=23$ chips, respectively. The analog modulating signal will be derived from the digital PRN chip sequence by replacing each 1 with a positive half-sine pulse of the form $\mathrm{m}(t)=\sin \pi f$ chip $t$ and each 0 with a negative half-sine pulse of the form $\mathrm{m}(t)=-\sin \pi f_{\text {chip }} t$. The composite code has a strong repeating $1010 \ldots$ pattern occasionally interrupted by a string of three or more 1's in a row. As a result, $\mathrm{m}(\mathrm{t})$ consists predominately of an alternating sequence of positive and negative half-sine 
pulses, thereby generating a sine wave with frequency $\mathrm{f}_{\mathrm{m}}=\mathrm{f}_{\text {chip }} / 2=1,034,733.544 \mathrm{~Hz}$. Since most of the power in the spectrum of $m(t)$ is concentrated at $f= \pm f_{m}$, the DLL is able to track the phase and frequency of the received code with very low jitter.

A diagram showing the overall structure of the uplink receiver is given in Figure 4 from Section 3 (Uplink Card). The receiver is a double-conversion super-heterodyne system with a $1^{\text {st }}$-IF frequency of $240 \mathrm{MHz}$ and a $2^{\text {nd }}-$ IF frequency of $2.5 \mathrm{MHz}$. The $2^{\text {nd }}$-IF actually consists of two parallel channels - a narrowband channel with a bandwidth of $2.5 \mathrm{MHZ} \pm 90 \mathrm{kHz}$ used by the PLL for tracking the phase of the residual carrier and a wideband channel with a bandwidth of $0-5 \mathrm{MHz}$ used by the RRC for tracking the time delay of the PRN ranging code. Both the PLL and the RRC use 10 Msps A/D converters to form digital samples of the narrow and wideband $2.5 \mathrm{MHz} 2^{\text {nd }}$-IF signals and both use digital processing algorithms to accomplish their tracking functions. The PLL adjusts the phase and frequency of the $1^{\text {st }}$ and $2^{\text {nd }}$ local oscillator (LO) signals such that the narrowband A/D samples occur at the zerocrossings and plus \& minus peaks of the narrowband 2.5 $\mathrm{MHz}$ sine wave. If the two $2^{\text {nd }}$-IF channels had equal phase since the phase shifts are not likely to be equal, the RRC is designed to execute an ADC clock adjustment procedure before tracking operations begin.

A block diagram of the RRC is given in Figure 16. With the exception of the A/D converter, the system is implemented entirely on one Actel RT54SX72S FPGA. The I and $\mathrm{Q}$ outputs of the demultiplexer will be $I_{k}=A \cos \left[\beta \mathrm{m}\left(t_{k}\right)\right]$ and $Q_{k}=A \sin \left[\beta \mathrm{m}\left(t_{k}\right)\right]$ respectively, where $A$ is the amplitude of the $2.5 \mathrm{MHz}$ phase modulated signal and $\beta$ is the modulation index. With $\beta=0.8$ radians, these two functions can be approximated as $I_{k} \approx A \mathrm{~J}_{0}(\beta)$ and $Q_{k}=2 A \mathrm{~J}_{1}(\beta) \mathrm{m}\left(t_{k}\right)$, respectively, where $\mathrm{J}_{0}(\beta)$ and $\mathrm{J}_{1}(\beta)$ are zero and first-order Bessel functions of the first kind. The I samples are therefore a measure of signal amplitude whereas the $Q$ samples provide the $\mathrm{m}(\mathrm{t})$ modulation function.

The $\mathrm{Q}$ samples are provided as input to a $2^{\text {nd }}$-order DLL that synchronizes the phase and frequency of the local code generator with the received uplink ranging code. The loop has three tracking bandwidths $-4 \mathrm{~Hz}$ for signal acquisition, $1 \mathrm{~Hz}$ for strong signal tracking, and $0.25 \mathrm{~Hz}$ for weak signal tracking. The loop is a discrete-time digital control system

fs

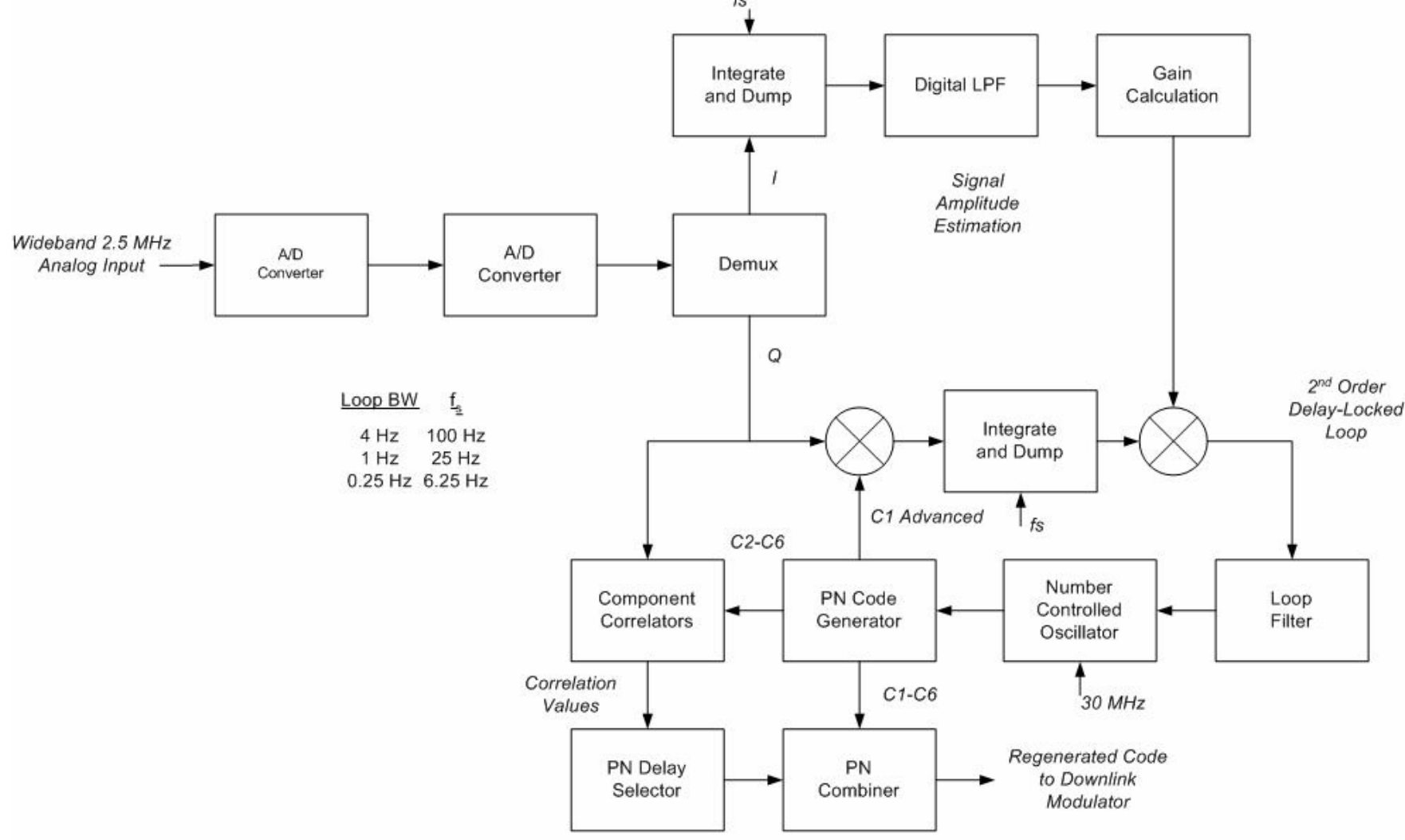

Figure 16 Regenerative Ranging Block Diagram

shift at $2.5 \mathrm{MHz}$, then the wideband $\mathrm{A} / \mathrm{D}$ samples would be with a phase detector, loop filter, number controlled oscillator, and PRN code generator. Phase error between 
the received uplink code and the local code generator is formed by multiplying the received $\mathrm{Q}$ samples by a halfchip advanced version of the local $\mathrm{C}_{1}$ code and integrating the resulting product for a period of 10,40 , or 160 milliseconds depending on the current loop operating bandwidth. Since the gain of the phase detector is proportional to signal amplitude, the loop has a provision for inserting a compensating gain function which varies inversely with signal amplitude in discrete power-of-2 steps. This function is accomplished by integrating and smoothing the I-samples and using the resulting values to generate an index for a power-of-2 gain table.

The DLL assures that the $\mathrm{C}_{1}$ component of the local code generator is aligned with the $\mathrm{C}_{1}$ component of the received ranging code and that the chip edges of the other component generators $\left(\mathrm{C}_{2}-\mathrm{C}_{6}\right)$ are also properly aligned. However since there are more than two chips in each of these components, a cyclical shift operation is generally required to properly align the component codes within the corresponding shift register generators. This operation is accomplished by multiplying and accumulating the received Q samples with each bit of each shift register, and selecting the shift position that generates the largest correlation product. Once these decisions have been made, the regenerated composite code is formed and passed on to the downlink modulator.

The tracking accuracy of the RRC is a function of the amplitude of the received RF signal and the tracking bandwidth. At low power levels, the tracking error is dominated by thermal noise whereas quantization errors due to finite bit widths dominate at the higher power levels. Figure 17 shows the predicted accuracy as a function of RF signal level and tracking bandwidth.

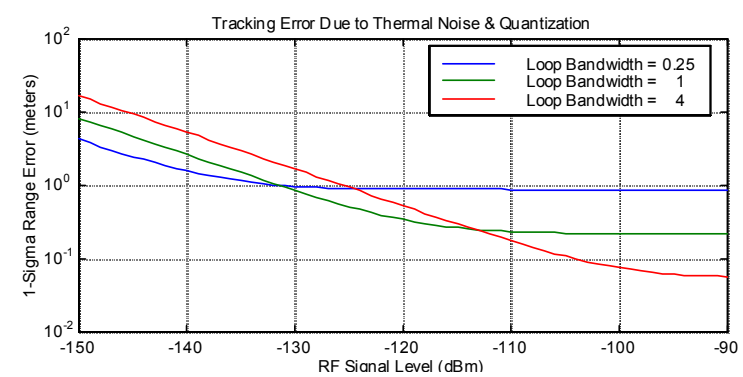

Figure 17 Tracking Error due to Thermal Noise and Quantization

\section{SUMMARY}

The RF Telecommunications System for the New Horizons Mission to Pluto has been described. The system includes a number of advanced capabilities: a low-power digital receiver, a card-based transceiver within an IEM, an ultrastable oscillator for precise frequency stability for the uplink radio science experiment, a noncoherent Doppler tracking capability, and the first planned operational use of regenerative ranging. Development of the system is nearing completion and flight fabrication of many system components has begun.

We are grateful to the NASA's Office of Space Science and the New Horizons Project Office for their support throughout the development of this system, in particular Tom Coughlin (program manager), Dave Kusnierkiewicz (mission systems engineer), and Chris Hersman (spacecraft systems engineer). Bob Bokulic and Matt Reinhart figured large in the early design of the system and have been valued counsel since. In addition, many engineers have worked and continue to work to bring the flight system together. In particular we would like to acknowledge the efforts of Laurel Funk, Rich DeBolt, Chris Fry, Steve Fortney, Paul Marth, Ballard Smith, and Michael Vincent.

\section{REFERENCES}

[1] R. Wohlleben, H. Mattes, O. Lochner, "Simple Small Primary Feed for Large Opening Angles and High Aperture Efficiency," Electronic Letters, 8, 474-476, 21 Sep 1972

[2] J. R. Jensen, R. S. Bokulic, "Experimental Verification of Noncoherent Doppler Tracking at the Deep Space Network", IEEE Transactions on Aerospace and Electronic Systems, 36, 4 (Oct 2000) 1401-1406

[3] J. R. Jensen, et al., "In-flight CONTOUR Radiometric Performance," 2003 IEEE Aerospace Conference, Big Sky, MT, March, 2003

[4] J. Berner, et al., "Regenerative Pseudo-Noise Ranging for Deep-Space Applications," TMO Progress Report 42137, Jet Propulsion Laboratory, Pasadena, CA, May 15, 1999 


\section{BIOGRAPHIES}

Chris DeBoy is the RF Telecommunications lead engineer

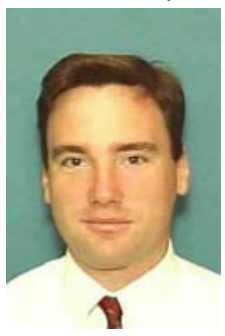

for the New Horizons mission. He received his BSEE from Virginia Tech in 1990, and the MSEE from the Johns Hopkins University in 1993. Prior to New Horizons, he led the development of a deep space, $X$-Band flight receiver for the CONTOUR mission and of an S-Band flight receiver for the TIMED mission. He remains the lead $R F$ system engineer for the TIMED and MSX missions. He has worked in the $R F$ Engineering Group at the Applied Physics Laboratory since 1990, and is a member of the IEEE.

Chris. Haskins is the lead engineer for the New Horizons

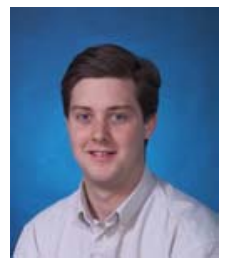
uplink receiver. He received a B.S. and M.S. from Virginia Tech in 1997 and 2000, both in electrical engineering. He joined the Johns Hopkins University Applied Physics Laboratory (APL) Space Department in 2000, where he has designed RF/Microwave, analog, and mixed-signal circuitry and subsystems in support of the CONTOUR, STEREO, and MESSENGER spacecraft. He also served as the lead engineer for the development of $R F$ ground support equipment for the CONTOUR spacecraft. Prior to working at APL, Mr. Haskins designed low cost commercial transceivers at Microwave Data Systems

Tom Brown (MSEE, BSEE from Clarkson University) is a

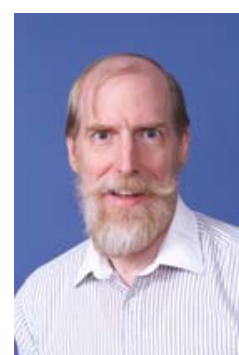
member of the Senior Professional Staff at Applied Physics Laboratory in the RF Engineering Group. During his 26 years career, mostly at General Electric and Ericsson, he has delved into various technologies -among them wireless communication, broadband, TCP/IP, security, IC design, lighting, video display, electron optics- and holds 18 patents. He is currently a member of IEEE, and the Biomedical Engineering group.

Ron Schulze is currently the lead antenna engineer for the

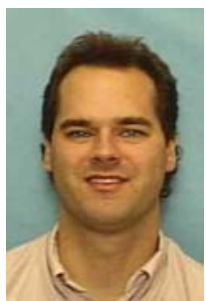
New Horizon mission. He received his B.S. from Virginia Tech in 1989, and M.S. from the Ohio State University in 1991, both in electrical engineering. He started his career at APL in 1991 in the Fleet Systems Department, where he performed propagation analysis studies related to lowelevation radar issues. He joined APL's Space Department in 1997, where he has designed planar micro-strip antennas and equatorial antennas for spin stabilized platforms. Mr. Schulze is also interested in technology development of lightweight inflatable reflector antennas. Prior to working at APL, Mr. Schulze worked at COMSAT Laboratories.

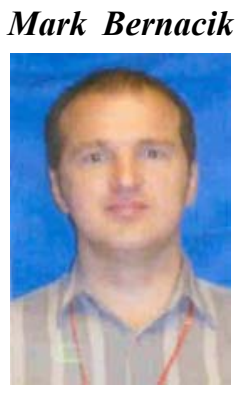

was born in Poland in 1960. He studied electrical engineering at Silesian Technical University in Gliwice, Poland. He received his BSEE from the University of Missouri/Columbia in 1989 and graduate work at the University of Kansas. He worked in industry (e.g., Oak Frequency Control (now Corning), Ericsson, Nokia, and Thales) primarily in $R F$ and analog circuit design for crystal oscillators, VCOs, PLL frequency synthesizers, radio receivers/transmitters and related circuits. At the Applied Physics Lab, he is a Lead Engineer for Ultra Stable Oscillator (USO) for the New Horizons mission to Pluto.

Bob Jensen (M'92) received a B.A. from Cornell College in

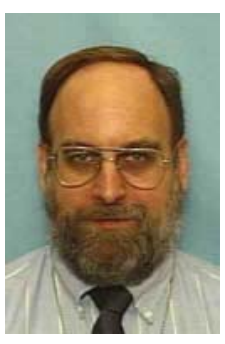
Mt. Vernon, Iowa, in 1973, and the Ph.D. in physical chemistry from the University of Wisconsin, Madison, in 1978. He joined The Johns Hopkins University Applied Physics Laboratory in 1978 and worked on a variety of nonacoustic detection problems, principally involving radar performance analysis, signal processing algorithms, and rough surface scattering. In 1989, he joined the APL Space Department and has participated in the TOPEX altimeter pre-flight testing, the development and testing of algorithms for the beacon receiver on the MSX satellite, the NEAR telecommunications system, and was responsible for noncoherent Doppler aspects of the CONTOUR mission. He is a member of the APL Principal Professional Staff and the IEEE.

Dennis Duven received the B.S., M.S., and Ph.D. degrees in

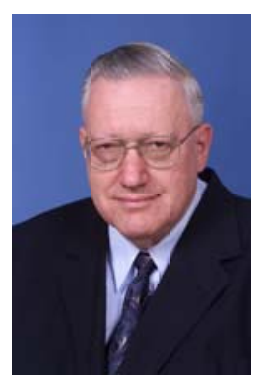
Electrical Engineering from Iowa State University in 1962, 1964, and 1971, respectively. He was responsible for coordinating and teaching the introductory Automatical Control Systems sequence at Iowa State from 1965-1973. Dr. Duven has been employed by the Space Department of the Johns Hopkins University Applied Physics Laboratory (APL) since 1973.

His responsibilities at APL have included analysis and design of the SATRACK I and II missile tracking systems, a Miss Distance Measurement System for the SDI Brilliant Pebbles Program, an autonomous GPS Navigation System 
for the NASA TIMED satellite, and the Regenerative Ranging System described in this paper.

Wes Millard is the lead engineer for the digital subsystems

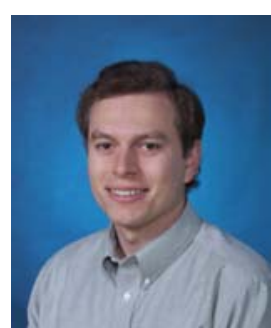
in the New Horizons uplink receiver. He received a B.S. in electrical engineering and in computer engineering in 1999, and a M.S.E. in electrical engineering in 2000, both at Johns Hopkins University. Since joining the APL Space Department in 2000, Wes has worked on the STEREO, MESSENGER, and New

Horizons programs where he has designed mixed signal circuitry and high efficiency DSP algorithms for FPGA implementation.

Stuart Hill is currently the lead mechanical engineer for the

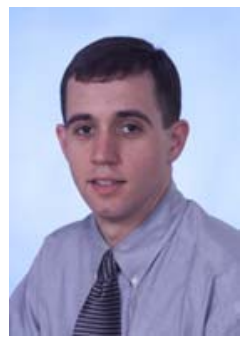
New Horizons antenna system. He received his B.S. from Texas A\&M University in 1999, and a M.E. from The Johns Hopkins University in 2003, both in mechanical engineering. He started his career at APL in 2000 in the Space Department, where he worked on the CONTOUR mission. Mr. Hill's interests include micro-mechanisms, lightweight spacecraft structures, and composite design. 